\title{
Asymmetric Vibrations and Chaos in Spherical Caps under Uniform Time-varying Pressure Fields
}

Giovanni larriccio ( $\square$ giovanni.iarriccio@unimore.it)

UNIMORE: Universita degli Studi di Modena e Reggio Emilia https://orcid.org/0000-0001-9323-8656

Antonio Zippo

UNIMORE: Universita degli Studi di Modena e Reggio Emilia

\section{Francesco Pellicano}

UNIMORE: Universita degli Studi di Modena e Reggio Emilia

\section{Research Article}

Keywords: Shells, spherical caps, vibrations, bifurcation, chaos

Posted Date: June 30th, 2021

DOl: https://doi.org/10.21203/rs.3.rs-657063/v1

License: (1) This work is licensed under a Creative Commons Attribution 4.0 International License.

Read Full License

Version of Record: A version of this preprint was published at Nonlinear Dynamics on November 10th, 2021. See the published version at https://doi.org/10.1007/s11071-021-07033-7. 
$4 \quad$ Iarriccio Giovanni

5 University of Modena and Reggio Emilia, 41121 Modena, Italy.

6 InterMech MoRe Centre, 41121 Modena, Italy.

7 ORCID id: 0000-0001-9323-8656

8 giovanni.iarriccio@unimore.it

9

10 Zippo Antonio

11 University of Modena and Reggio Emilia, 41121 Modena, Italy.

12 InterMech MoRe Centre, 41121 Modena, Italy.

13 ORCID id: 0000-0001-6206-2619

14

15 Pellicano Francesco

16 University of Modena and Reggio Emilia, 41121 Modena, Italy.

17 InterMech MoRe Centre, 41121 Modena, Italy.

18 ORCID id: 0000-0003-2465-6584

19

20

23 N. of pages: 32

24 N. of figures: 11

25 N. of tables: 1

26 


\section{Abstract}

28 This paper presents a study on nonlinear asymmetric vibrations in shallow 29 spherical caps under pressure loading. The Novozhilov's nonlinear shell theory is 30 used for modelling the structural strains. A reduced-order model is developed 31 through the Rayleigh-Ritz method and Lagrange equations. The equations of 32 motion are numerically integrated using an implicit solver. The bifurcation 33 scenario is addressed by varying the external excitation frequency. The 34 occurrence of asymmetric vibrations related to quasi-periodic and chaotic motion 35 is shown through the analysis of time histories, spectra, Poincaré maps, and 36 phase planes.

37

38 Keywords

39 Shells, spherical caps, vibrations, bifurcation, chaos.

40 
42 Thin-walled structures like plates, panels, shells, and caps are important structural 43 elements in Engineering; their applications can be found in Civil Engineering 44 (roofs, vaults, tensile structures), Aerospace (airplanes, missiles and rockets);

45 Mechanics (membrane based microsensors and energy harvesters).

46 These structures are strong and lightweight at the same time, but they are

47 extremely sensitive to perturbations, present a complicated instability behaviors 48 and are very difficult to model. They could buckle under the action of critical 49 loads, following sub-critical post-buckling paths; moreover, they can exhibit non50 linear dynamic phenomena, such as chaotic vibrations, when the amplitude of 51 vibration is moderately large.

52 Nowadays, many theories and simplified models are available for studying shell 53 systems, even in the presence of fluid-structure interaction or thermal fields. 54 Nevertheless, new challenges come from the new frontiers of the Engineering, 55 which asks for even more reliable models where complicating effects are taken 56 into account for exploiting the nonlinearities: for example, phenomena such as 57 multi-stability or the pull-in, can be desired features through which designers can 58 achieve structural optimization and develop high performance devices.

59 A short literature review is reported here for introducing the reader to the most 60 important and recent scientific contributions to the study of thin walled structures, 61 with a specific focus to spherical caps dynamics.

62 Concerning the elastic stability of shells, buckling problems are classified into:

63 Static buckling when loads are applied extremely slowly; Dynamic buckling when 64 the loads are suddenly applied (step loads).

65 From a literature review, there is a discrepancy between experimental data and 66 theoretical results. The primary sources of inconsistencies, that lead to an 67 experimental lower buckling load than the one theoretically predicted, are (i) the 68 high sensitivity of shells to geometric imperfections and non-uniform material 69 distribution, and (ii) the post-buckling behavior is strongly affected by 70 nonlinearities.

71 Let us first focus on the static instability of the spherical caps under an external 72 pressure load. Krenze and Kiernan [1] showed the importance of producing high 73 quality specimens for performing experimental tests. In the same period, Huang 
74 [2] and Weinitsche [3] used Margurre's theory with possibility of having non-

75 symmetric buckling. They showed how, for deeper caps, the wavelength of the

76 buckling modes was higher compared to shallow caps, and numerical results

77 agreed with the experimental ones available at that time. These results were

78 experimentally confirmed by Yamada et al. [4] two decades later.

79 The role of geometric imperfections on critical static loads of caps was

80 investigated in Refs. [5,6]. Results pointed out how the shape of the geometric

81 imperfection affects the decrement of the critical buckling load; often the snap-

82 through phenomenon disappeared due to imperfections, and continuous and stable

83 buckling paths were shown by the pressure-deflection diagrams.

84 Since the measurement of imperfections it is not always possible for large scale

85 applications, NASA proposed an empirical formula based on the lower envelope

86 of a series of experimental data [7]. Nowadays, the specimens quality is higher

87 and other techniques have been proposed for improving the NASA empirical

88 formula[8,9].

89 A further reduction of the load-carrying capacity can be observed when the time

90 dependency of the load is considered, i.e. in the case of dynamic buckling.

91 Lock et al. [10] experimentally analyzed the buckling of shallow domes under a

92 pressure-step loading. They discussed the difference between "direct" and

93 "indirect" snapping phenomena. The direct snapping is a catastrophic

94 phenomenon and involves only symmetric vibrations; conversely, the indirect

95 snapping occurs after a transient and the contribution of the non-symmetric modes

96 is not negligible after the snapping.

97 Stricklin et al. [11] used nonlinear Novozhilov's theory for investigating the static

98 stresses in shells of revolution and improved their model for studying the dynamic

99 buckling in Ref. [12]. The equilibrium equations were obtained through

100 Castigliano's theorem. Numerical results were compared with experimental ones,

101 and an excellent agreement was proved. The dynamic model was derived

102 employing of the Lagrange equations by considering only axisymmetric modes,

103 and the results confirmed the previous analyses [13,14].

104 Ball and Burt [15] numerically investigated the dynamic buckling of clamped

105 shallow spherical caps under symmetric and nearly-symmetric step pressure loads.

106 Asymmetric modes were considered, and the buckling load of geometrically

107 perfect structures of different shallowness was given. 
108 The asymmetric dynamic buckling of shallow spherical caps was investigated 109 even by Akkas [16], who showed that the asymmetric buckling under step 110 pressure load results in cusps in phase-plane diagrams.

111 Further results concerning the dynamic buckling of imperfect caps can be found in 112 Refs. [17-19], where the possibility of having plastic deformations was 113 considered as well.

114 In the framework of spherical caps under harmonic loads, the literature is not as 115 vast as for the buckling. Reasons must be sought in the fact that: (i) spherical caps 116 are a particular case of doubly curved shells, they are modeled through equations 117 that are more complex with respect to plates and cylindrical shells; (ii) the high 118 computational cost related to the numerical integration of the equations of motion 119 limited for long time the analysis to low dimensional models and axisymmetric 120 vibrations.

121 Using a theory proposed by $\mathrm{Yu}$ [20], Grossman et al. [21] investigated the 122 axisymmetric nonlinear vibrations of shallow spherical caps with different 123 boundary conditions. This study compared flat plates to curved caps, and the 124 results pointed out the transition from hardening to softening nonlinearity when 125 the surface curvature is increased.

126 Evensen and Iwanovsky [22] were the first to perform both analytically and 127 experimental analyses on shallow spherical caps under a combination of static and 128 sinusoidal external pressure loads. The analytical model was based on the 129 Marguerre's nonlinear shell theory. Axisymmetric deflections and uniform load 130 distribution were considered. A detailed scheme of the experimental setup was 131 reported and discussed. Numerical results concerning free vibrations were in 132 excellent agreement with experiments. Unfortunately, differences were shown in 133 several nonlinear forced cases. Such discrepancies were mainly attributed to the 134 interaction between static and dynamic loads, and to the asymmetric vibrations 135 observed during the experiments.

136 Yasuda and Kushida [23] studied the forced vibration of caps under harmonic 137 point loads. The activation of subharmonic motion due to internal resonances was 138 observed. In order to validate the numerical model, experiments were performed 139 on a bent circular plate clamped at its edges. The structure was loaded by a 140 concentrated force induced by two electric magnets, and experimental results 141 agreed with the numerical ones. 
142 The axisymmetric vibrations of pre-loaded shallow spherical caps were 143 investigated by Gonçalves [24,25] and Soliman and Gonçalves [26]. For obtaining 144 a reduced-order model (ROM), the Galerkin method was considered. The 145 displacement fields were expanded by using the Bessel functions, and the 146 resulting equations of motion were solved through the Newton-Rapson method.

147 Results showed a strong influence of geometric imperfections and their spatial 148 shape. Softening nonlinearity can be turned to hardening by imposing a suitable 149 initial imperfection of a given shape and amplitude, as shown by the reported 150 backbone curves. Moreover, assuming the excitation frequency as a control 151 parameter, the bifurcation analysis pointed out the existence of period-doubling 152 cascades and chaotic oscillations. The onset of such phenomena is due to energy 153 given by the harmonic pressure to the shell, which leads to multiple back-and154 forth jumps between potential wells.

155 Thomas et al. [27,28] studied the response of a free-edge shallow spherical cap 156 under harmonic excitation. Using the multiple-scale perturbation method, results 157 showed that, having integer or quasi-integer ratio between natural frequencies is 158 not a sufficient condition for having internal resonances activation. This is due to 159 the body symmetry, which leads to the canceling of some nonlinear coefficient in 160 the ODEs. Experiments were carried out by forcing the specimen using an 161 electromagnetic coil. The occurrence of an internal resonance between two 162 conjugate asymmetric modes and one axisymmetric mode (1:1:2) was proven, a 163 good qualitative fitting between theory and experiments was shown for small 164 forcing amplitude.

165 Touzè et al.[29,30] used the nonlinear normal modes approach (NNMs) for 166 predicting the trend of nonlinearity for each mode as a function of the spherical 167 cap geometric aspect ratios. In particular, the transition from hardening to 168 softening nonlinearity was addressed.

169 Chaotic vibrations in shallow shells with circular planform were investigated by 170 Krysko et al. [31]: the role of size-dependent parameters on vibrations of nano 171 shells were analyzed. The system of PDE was reduced using a finite difference 172 method (FDM), and the resulting system was solved through a Runge-Kutta 173 scheme. By comparing Fourier's spectra, Poincaré maps, Lyapunov exponents, 174 and Morlet wavelet, the authors showed that, considering the size-effect shells 
175 exhibit regular vibrations whereas with the same load conditions neglecting the

176 size-effect one obtains chaotic vibrations.

177 The present work aims to address to some questions arisen recently in Ref.[32] on

178 pressure loaded spherical caps, where the limits of axisymmetric models were

179 shown using continuation techniques. Here the Novozhilov's geometrically

180 nonlinear theory is considered. For the analysis of the linearized equations, the

181 Rayleigh-Ritz approach is considered to obtain the mode shapes in a semi-

182 analytical way. Lagrange equations are used for reducing the system of nonlinear

183 partial differential equations, PDEs, to a system of ordinary differential equations,

184 ODEs. A bifurcation analysis of is carried out by directly integrating the equations

185 of motion. Results are presented and discussed with the help of bifurcation

186 diagrams and other useful tools, such as Poincaré maps and Fourier's spectra. The

187 superimposition of a static and a dynamic pressure yields to non-periodic and

188 chaotic oscillation related to the activation of asymmetric modes.

\section{Problem Formulation}

191 A spherical cap having radius $R$, base radius $a$, cap height $S$, and thickness $h$,

192 is considered, see Fig. 1(a-c). A spherical coordinate system $(O ; \varphi, \vartheta, z)$ is

193 centered at the top of the cap $O$. The curvilinear coordinates $(\varphi, \vartheta)$ identify a

194 point $P$ of the shell middle surface, $z$ is the radial distance of a generic point of

195 the shell from the middle surface. Three displacement fields, meridional

$196 u(\eta, \vartheta, t)$, circumferential $v(\eta, \vartheta, t)$, and radial $w(\eta, \vartheta, t)$, describe the deformed

197 configuration of the middle surface; $t$ is the time variable.

198 Limiting the analysis to shallow spherical caps, the Lamé parameters of the 199 undeformed middle surface are $A_{1}=R$ and $A_{2} \cong \varphi_{b} \cdot \eta$; where $\eta=\varphi / \varphi_{B}$ is the

200 meridional non-dimensional coordinate.

201 For describing the relationships between strains and displacements, the 202 Novozhilov's nonlinear shell theory [33] is considered. Such theory is based on 203 the Kirchhoff-Love hypothesis, which states that: (i) the shell is thin $h \ll R$ and $204 h \ll a$, (ii) strains, (iii) transverse normal stresses are small, and (iv) the normal to 205 the undeformed middle surface remains normal after deformation, and no 
206 thickness stretching occurs. The hypothesis of small displacements is relaxed in 207 the nonlinear analysis.

208

(a)

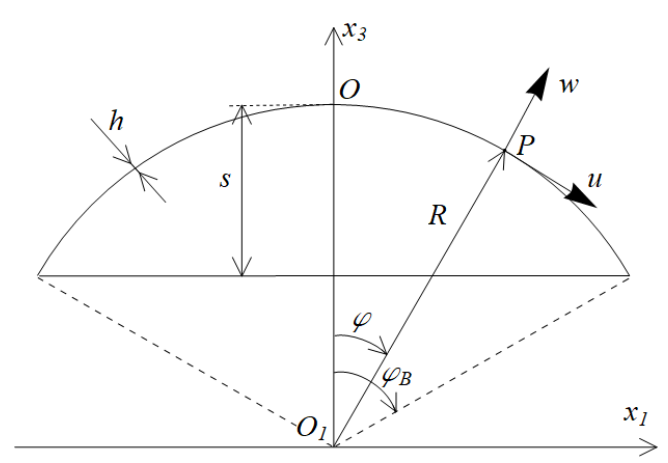

(b)

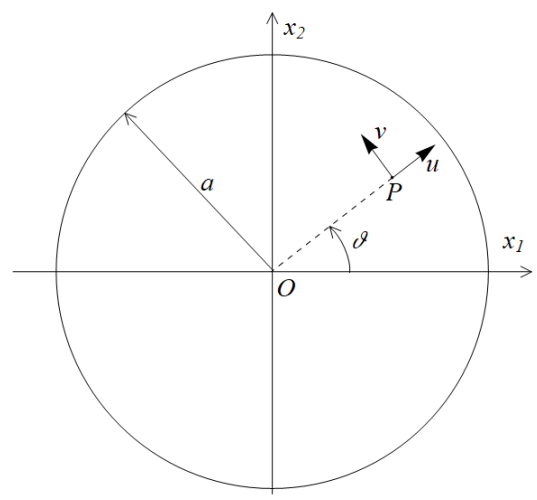

(c)

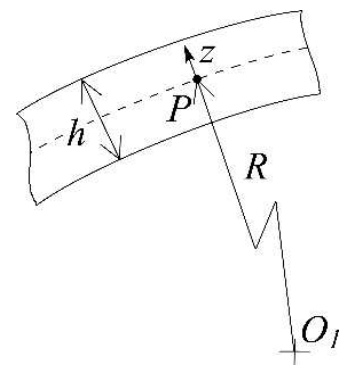

209 Fig. 1. Spherical cap geometry and coordinate system: (a) cross-section view, (b) top view, 210 and (c) breakout-section view.

212 Because of the aforementioned hypothesis, the strains $\hat{\varepsilon}_{i}, \hat{\gamma}_{i j}$ at an arbitrary point 213 of the cap linearly vary along the thickness; moreover, the plane-stress hypothesis 214 is considered. The strains are given by:

215

$$
\begin{aligned}
& \hat{\varepsilon}_{\eta}=\varepsilon_{\eta}+z \cdot k_{\eta}, \\
& \hat{\varepsilon}_{\vartheta}=\varepsilon_{\vartheta}+z \cdot k_{\vartheta}, \\
& \hat{\gamma}_{\eta \vartheta}=\gamma_{\eta \vartheta}+z \cdot k_{\eta \vartheta},
\end{aligned}
$$

217 where $\varepsilon_{\eta}, \varepsilon_{\vartheta}, \gamma_{\eta \vartheta}$ are the middle surface strains, $k_{\eta}, k_{\vartheta}$, and $k_{\eta \vartheta}$ are the changes 218 in curvatures and torsion of the middle surface of the shell, which depend on the 219 middle surface displacement fields through the following relationships: 


$$
\begin{aligned}
& \varepsilon_{\eta}=e_{11}+\frac{1}{2}\left(e_{11}^{2}+e_{12}^{2}+e_{13}^{2}\right), \\
& \varepsilon_{\vartheta}=e_{22}+\frac{1}{2}\left(e_{21}^{2}+e_{22}^{2}+e_{23}^{2}\right), \\
& \gamma_{\eta \vartheta}=e_{12}+e_{21}+e_{11} e_{21}+e_{12} e_{22}+e_{12} e_{23}, \\
& k_{\eta}=-\frac{1}{A_{1}} \frac{\partial e_{13}}{\varphi_{b} \partial \eta}+\frac{e_{11}+e_{22}}{R}, \\
& k_{\vartheta}=-\frac{1}{A_{1} A_{2}} \frac{\partial A_{2}}{\varphi_{b} \partial \eta} e_{13}+\frac{1}{A_{1}} \frac{\partial e_{23}}{\varphi_{b} \partial \eta}+\frac{e_{11}+e_{22}}{R}, \\
& k_{\eta \vartheta}=-\frac{1}{A_{2}} \frac{\partial e_{13}}{\partial \vartheta}-\frac{1}{A_{1}} \frac{\partial e_{23}}{\varphi_{b} \partial \eta}+\frac{1}{A_{1} A_{2}} \frac{\partial A_{2}}{\varphi_{b} \partial \eta} e_{23} .
\end{aligned}
$$

222 where the strain components $e_{i j}$ are:

223

$$
\begin{aligned}
& e_{11}=\frac{1}{A_{1}} \frac{\partial u}{\varphi_{b} \partial \eta}+\frac{w}{R}, \\
& e_{12}=\frac{1}{A_{2}} \frac{\partial u}{\partial \vartheta}-\frac{1}{A_{1} A_{2}} \frac{\partial A_{2}}{\varphi_{b} \partial \eta} v, \\
& e_{13}=-\frac{u}{R}+\frac{1}{A_{1}} \frac{\partial w}{\varphi_{b} \partial \eta}, \\
& e_{21}=\frac{1}{A_{1}} \frac{\partial v}{\varphi_{b} \partial \eta}, \\
& e_{22}=\frac{1}{A_{1} A_{2}} \frac{\partial A_{2}}{\varphi_{b} \partial \eta} u+\frac{1}{A_{2}} \frac{\partial v}{\partial \vartheta}+\frac{w}{R}, \\
& e_{23}=-\frac{v}{R}+\frac{1}{A_{2}} \frac{\partial w}{\partial \vartheta} .
\end{aligned}
$$

225 Considering an elastic linear, homogeneous and isotropic continuum, one can use 226 the Hooke's law, i.e. the following stress-strain relationships: 


$$
\left(\begin{array}{c}
\hat{\sigma}_{\eta} \\
\hat{\sigma}_{\eta} \\
\hat{\tau}_{\eta \vartheta}
\end{array}\right)=\left[\begin{array}{ccc}
\frac{E}{1-v^{2}} & \frac{v E}{1-v^{2}} & 0 \\
\frac{v E}{1-v^{2}} & \frac{E}{1-v^{2}} & 0 \\
0 & 0 & \frac{E}{2(1+v)}
\end{array}\right]\left(\begin{array}{c}
\hat{\varepsilon}_{\eta} \\
\hat{\varepsilon}_{\vartheta} \\
\hat{\gamma}_{\eta \vartheta}
\end{array}\right)
$$

228

229 where $E$ and $v$ are the Young's modulus and the Poisson's ratio, respectively.

230 By considering the strains (1.a-d) and the stresses (4.a-b), the elastic strain energy $231 U_{S}[34]$ of a thin shallow spherical cap is given by

232

$$
\begin{aligned}
U_{S}= & \frac{1}{2} \int_{-h / 2}^{h / 2} \int_{\eta_{0}}^{1} \int_{0}^{2 \pi}\left(\hat{\sigma}_{\eta} \hat{\varepsilon}_{\eta}+\hat{\sigma}_{\vartheta} \hat{\varepsilon}_{\vartheta}+\hat{\tau}_{\eta \vartheta} \hat{\gamma}_{\eta \vartheta}\right) A_{1} A_{2} \varphi_{b} d \vartheta d \eta \\
= & \frac{1}{2} \frac{E h}{1-v^{2}} \int_{\eta_{0}}^{1} \int_{0}^{2 \pi}\left(\varepsilon_{\eta}^{2}+\varepsilon_{\vartheta}^{2}+2 v \varepsilon_{\eta} \varepsilon_{\vartheta}+\frac{1-v}{2} \gamma_{\eta \vartheta}^{2}\right) A_{1} A_{2} \varphi_{b} d \vartheta d \eta+ \\
& \frac{1}{2} \frac{E h^{3}}{12\left(1-v^{2}\right)} \int_{\eta_{0}}^{1} \int_{0}^{2 \pi}\left(k_{\eta}^{2}+k_{\vartheta}^{2}+2 v k_{\eta} k_{\vartheta}+\frac{1-v}{2} k_{\eta \vartheta}^{2}\right) A_{1} A_{2} \varphi_{b} d \vartheta d \eta
\end{aligned}
$$

233

234 while, the kinetic energy $T_{S}$, under the hypothesis of negligible rotary inertia [34],

235 is given by

236

$$
T_{S}=\frac{1}{2} \rho_{S} h \int_{\eta_{0}}^{1} \int_{0}^{2 \pi}\left(\dot{u}^{2}+\dot{v}^{2}+\dot{w}^{2}\right) A_{1} A_{2} \varphi_{b} d \vartheta d \eta
$$

238 where $\rho_{S}$ is the material mass density, $\eta_{0}$ is the half opening angle of a hole 239 assumed a the cap pole for avoiding the singularity due to the spherical reference 240 system [35].

\subsection{Approximate eigenfunctions}

243 In order to develop a ROM for studying the cap nonlinear dynamics, in this

244 section the eigenfunctions of the linearized operator are obtained through the 245 Rayleigh-Ritz approach [36]. 
246 In the present study, clamped boundary conditions are considered at the circular 247 edge

$$
u=v=w=\frac{\partial w}{\partial \eta}=0 \quad \text { for } \quad \eta=1
$$

248 while, no boundary conditions are considered at the cap pole.

249 The Rayleigh-Ritz approach requires that a trial function set respects the 250 geometric boundary conditions only [37]; on the other hand, the stress-free 251 boundary conditions at the cap pole (where the small hole is present) are 252 neglected.

253 The generic mode of vibration can be described by considering three displacement 254 fields $u(\eta, \vartheta, t), v(\eta, \vartheta, t)$, and $w(\eta, \vartheta, t)$, which obey to the same time law $f(t)$, 255 i.e. the variable separation can be considered:

256

$$
\begin{aligned}
& u(\eta, \vartheta, t)=U(\eta, \vartheta) \cdot f(t), \\
& v(\eta, \vartheta, t)=V(\eta, \vartheta) \cdot f(t), \\
& w(\eta, \vartheta, t)=W(\eta, \vartheta) \cdot f(t) .
\end{aligned}
$$

$258 U(\eta, \vartheta), V(\eta, \vartheta)$, and $W(\eta, \vartheta)$ are spatial functions denoting the mode shapes i.e.

259 eigenfunctions.

260 The eigenfunctions are now discretized using a linear combination of functions.

261 Legendre polynomials are considered in the meridional direction and 262 trigonometric functions are assumed in the circular direction.

$$
\begin{aligned}
U(\eta, \vartheta)= & \sum_{m=0}^{M_{u}} \sum_{n=0}^{N} \tilde{U}_{m, n} P_{m}^{*}(\eta) \cos (n \vartheta), \\
V(\eta, \vartheta)= & \sum_{m=0}^{M_{v}} \tilde{V}_{m, 0} P_{m}^{*}(\eta)+ \\
& \sum_{m=0}^{M_{v}} \sum_{n=1}^{N} \tilde{V}_{m, n} P_{m}^{*}(\eta) \sin (n \vartheta), \\
W(\eta, \vartheta)= & \sum_{m=0}^{M_{w}} \sum_{n=0}^{N} \tilde{W}_{m, n} P_{m}^{*}(\eta) \cos (n \vartheta) .
\end{aligned}
$$


265 where $P_{m}^{*}(\eta)=P_{m}(2 \eta-1)$ is the $m$-th Legendre polynomial of the first kind

266 shifted in the domain $\eta \in[0,1] ; m$ is related to the number of meridional

267 wavelength; $n$ is the number of nodal diameters.

268 Because of the axial symmetry, spherical caps exhibit conjugate modes, called

269 driven and companion mode shapes or conjugate modes [38,39]. These modes 270 have the same natural frequency and shape, but the displacement fields are 271 angularly shifted of $\pi / 2 n$. Conjugate modes describe standing waves, but 272 circumferential travelling waves could arise when nonlinear mode coupling 273 occurs [40-42]. Therefore, companion modes should be considered when a 274 nonlinear analysis is carried out.

275

$$
\begin{aligned}
& U(\eta, \vartheta)=\sum_{m=0}^{M_{u}} \sum_{n=1}^{N} \tilde{U}_{m, n} P_{m}^{*}(\eta) \sin (n \vartheta), \\
& V(\eta, \vartheta)=\sum_{m=0}^{M_{v}} \sum_{n=1}^{N} \tilde{V}_{m, n} P_{m}^{*}(\eta) \cos (n \vartheta), \\
& W(\eta, \vartheta)=\sum_{m=0}^{M_{w}} \sum_{n=1}^{N} \tilde{W}_{m, n} P_{m}^{*}(\eta) \sin (n \vartheta) .
\end{aligned}
$$

276

277 It is worth noting that asymmetric modes are not associated to multiple

278 eigenvalues, therefore, they have not companion modes.

279 By imposing the set of boundary conditions (7) to the discretized eigenfunctions, 280 a system of algebraic equations is obtained:

281

$$
\begin{aligned}
& \sum_{m=0}^{M_{u}} \tilde{U}_{m, n} P_{m}^{*}(\eta)=0, \\
& \sum_{m=0}^{M_{v}} \tilde{V}_{m, n} P_{m}^{*}(\eta)=0, \\
& \sum_{m=0}^{M_{w}} \tilde{W}_{m, n} P_{m}^{*}(\eta)=0, \quad \text { for } \quad \eta=1 \\
& \sum_{m=0}^{M_{w}} \tilde{W}_{m, n} \frac{\partial}{\partial \eta} P_{m}^{*}(\eta)=0,
\end{aligned}
$$

283 The solution of this linear system allows to express $\left(\tilde{U}_{0, n}, \tilde{V}_{0, n}, \tilde{W}_{0, n}, \tilde{W}_{1, n}\right)$ in terms 284 of the remaining coefficients $\left(\tilde{U}_{m, n}, \tilde{V}_{m, n}, \tilde{W}_{m, n}\right)$; the latter coefficients can be 
285 reordered in a vector $\tilde{\mathbf{q}}$ [43] with a number of elements equal to

$286 N_{\max }=\left(M_{u}+M_{v}+M_{w}+3-b\right)(N+1)$, where $b=4$ for a clamped circular cap [32].

287 Considering only the linear terms in the strain-displacement relations (2.a-f), the

288 eigenvalue problem for approximating the natural frequencies and mode shapes of 289 the structure is obtained by imposing the stationarity of the Rayleigh's quotient $290 R(\tilde{\mathbf{q}})=U_{S}(\tilde{\mathbf{q}}) / T_{S}^{*}(\tilde{\mathbf{q}})$, where $U_{S}(\tilde{\mathbf{q}})$ is the maximum potential energy during a

291 "modal" oscillation, and $T_{S}^{*}(\tilde{\mathbf{q}})=T_{S}(\tilde{\mathbf{q}}) / \omega^{2}$.

292

$$
\left(-\omega^{2} \mathbf{M}+\mathbf{K}\right) \tilde{\mathbf{q}}=\mathbf{0} .
$$

293

$294 \omega$ is the circular frequency of the harmonic motion; $\mathbf{M}$ and $\mathbf{K}$ are the mass matrix and the stiffness matrix of the discrete linearized system, respectively.

296 The $i$-th solution of equation (13), $\left(\omega^{(i)}, \tilde{\mathbf{q}}^{(i)}\right)$, gives the approximation of the $i$-th 297 natural frequency and mode shape, respectively.

298 To improve the results readability and the numerical accuracy, the approximated 299 mode shapes are normalized using the approach of Ref.[43], and the following 300 condition is sought $\max \left[\operatorname{abs}\left[U^{(i)}(\eta, \vartheta)\right], \operatorname{abs}\left[V^{(i)}(\eta, \vartheta)\right], \operatorname{abs}\left[W^{(i)}(\eta, \vartheta)\right]\right]=1$.

\subsection{Nonlinear vibrations}

303 Synchronous motion and small amplitude displacement hypotheses are now 304 relaxed, as well as the absence of external excitation.

305 In such conditions we cannot claim anymore that the vibration is harmonic or 306 periodic.

307 The approach used for analyzing the nonlinear dynamics of the cap is based on the 308 spectral theorem, i.e., taking advantage from the completeness of the 309 eigenfunctions calculated on the previous section, the displacement fields are 310 expanded as follows:

311

$$
\begin{aligned}
& u(\eta, \vartheta, t)=\sum_{i}^{M_{u, 1}} \sum_{j}^{N_{u}}\left[U_{i, j}^{(d)}(\eta, \vartheta) f_{u, i, j}^{(d)}(t)+U_{i, j}^{(c)}(\eta, \vartheta) f_{u, i, j}^{(c)}(t)\right] \\
& v(\eta, \vartheta, t)=\sum_{i}^{M_{v, 1}} \sum_{j}^{N_{v}}\left[V_{i, j}^{(d)}(\eta, \vartheta) f_{v, i, j}^{(d)}(t)+V_{i, j}^{(c)}(\eta, \vartheta) f_{v, i, j}^{(c)}(t)\right]
\end{aligned}
$$




$$
w(\eta, \vartheta, t)=\sum_{i}^{M_{w, 1}} \sum_{j}^{N_{w}}\left[W_{i, j}^{(d)}(\eta, \vartheta) f_{w, i, j}^{(d)}(t)+W_{i, j}^{(c)}(\eta, \vartheta) f_{w, i, j}^{(c)}(t)\right]
$$

313 where $d$ and $c$ are related to the driven and companion modes, respectively; $i$ and $j$

314 identify the number of meridional and circumferential wavelengths; $f_{k, i, j}^{(\cdot)}$ are the

315 time dependent unknown generalized coordinates.

316 For thin-walled bodies under external pressure load, two assumptions are common

317 in the literature: (i) the pressure is considered as a radial non-follower load; (ii)

318 the load distribution is applied to the middle surface [33].

319 The former approximation simplifies the numerical calculations and reduces the

320 numerical effort; however, it could underestimate the safety factor in structures

321 that undergo to large deflections. The latter assumption is a valid approximation

322 for thin shells and should be removed for thicker structures.

323 Considering a configuration-dependent pressure distribution that always acts 324 orthogonal to the surface (follower force distribution), the expression of the $j$-th 325 generalized force is given by Amabili and Breslavsky, where only the linear strain 326 terms are retained [44]

$$
\frac{\partial W_{p}}{\partial q_{j}} \cong \int_{\eta_{0}}^{1} \int_{0}^{2 \pi} p(t)\left[-\frac{\partial u}{\partial q_{j}} e_{12}-\frac{\partial v}{\partial q_{j}} e_{23}+-\frac{\partial w}{\partial q_{j}}\left(1+e_{11}+e_{22}\right)\right] A_{1} A_{2} \varphi_{b} d \vartheta d \eta
$$

329 The external pressure consists of a static and a dynamic component $330 p(t)=p_{s}+p_{d} \cos (\Omega t)$ is the external pressure. The pressure is positive when 331 inflates the structure.

332 Taking into account the full expression of the strains (2.a-f) and replacing them 333 into the energies and virtual work formulae, the equations of motion are derived 334 by the Lagrange equations

$$
\frac{d}{d t}\left(\frac{\partial T_{S}}{\partial \dot{q}_{j}}\right)+\frac{\partial U_{S}}{\partial q_{j}}=\frac{\partial W_{p}}{\partial q_{j}}, \quad \text { for } \quad j=1,2, \ldots, N_{d o f s}
$$

$337 N_{\text {dofs }}$ indicates the number of degrees of freedom of the nonlinear ODEs. Such set 338 could be rewritten into state-space form.

339 The set of nonlinear ODEs could be rewritten into the following first-order form: 


$$
\left\{\begin{array}{c}
\dot{\mathbf{q}}=\mathbf{y} \\
\dot{\mathbf{y}}=\mathbf{M}^{-1}\left[-\mathbf{C y}-\mathbf{K}_{N L} \mathbf{q}+\mathbf{p}_{s}+\mathbf{p}_{d} \cos (\Omega t)\right]
\end{array}\right.
$$

341

342 Note that $\mathbf{M}^{-1} \mathbf{C}=\operatorname{diag}\left(2 \zeta_{j} \omega_{j}\right)$, where $\zeta_{j}$ and $\omega_{j}$ are the damping ration and the

343 natural frequencies of the $j^{\text {th }}$ generalized coordinate; $\mathbf{p}_{s}$ and $\mathbf{p}_{d}$ are the

344 generalized force vectors due to the static and dynamic pressure, respectively; $\Omega$

345 is the frequency of the external excitation; $\mathbf{y}$ is the generalized velocity vector.

346 In the following analysis, the equations of motion are reduced to a 347 nondimensional form: the amplitudes are divided by the shell thickness; the time

348 is divided by the period of the first axisymmetric resonant mode.

$$
\hat{\mathbf{q}}=\mathbf{q} / h \quad \tau=\omega_{1,0} \cdot t
$$

350

351 The pressure is normalized through to the Zoelly's critical buckling pressure of a

352 complete, isotropic, and homogeneous sphere, see Ref. [45]

353

$$
p_{c r}=\frac{2 E}{\sqrt{3\left(1-v^{2}\right)}}\left(\frac{h}{R}\right)^{2}
$$

354

355 For the sake of completeness, the expression of the parameter $\lambda$ is here reported

356 due to its important meaning: $\lambda$ includes information on the thinness and the

357 shallowness of the investigated structure.

358

$$
\lambda=\sqrt[4]{12\left(1-v^{2}\right)} \cdot \frac{a}{\sqrt{R h}}
$$

\section{Numerical Results}

361 Consider a clamped shallow spherical cap, having a uniform thickness, made of

362 steel. Using the 38 dofs nonlinear model developed in Ref [32], the nonlinear

363 dynamic response of the cap under a time-varying harmonic pressure is 
364 investigated. For the sake of clarity, the linear mode shapes retained into the

365 nonlinear ROM are listed in Table 1:it must be noted that both driven and

366 companion vibration modes have been included for the asymmetric mode shapes

$367(n=0)$.

Table 1 - Normalized natural frequencies and mode shapes considered into the nonlinear reduced-order model [32].

\begin{tabular}{|l|l|l|l|}
\hline$\omega_{m, n} / \omega_{1,0}$ & $\boldsymbol{m}$ & $\boldsymbol{n}$ & Modal displacement field \\
\hline 1.0000 & 1 & 0 & $w, u$ \\
\hline 1.1052 & 1 & 2 & $w, v, u$ \\
\hline 1.3030 & 2 & 0 & $w, u$ \\
\hline 1.6838 & 1 & 4 & $v$ \\
\hline 1.9650 & 2 & 2 & $w, v, u$ \\
\hline 2.0695 & 3 & 0 & $w, u$ \\
\hline 2.4869 & 2 & 4 & $v$ \\
\hline 2.6661 & 3 & 2 & $w, v, u$ \\
\hline 3.4519 & 4 & 0 & $w, u$ \\
\hline 4.1146 & 3 & 4 & $v$ \\
\hline 5.3040 & 5 & 0 & $w, u$ \\
\hline 7.3148 & 4 & 4 & $v$ \\
\hline 7.7524 & 6 & 0 & $w, u$ \\
\hline
\end{tabular}

372 The geometrical and structural data are listed as follows: $R=0.8 m, h=R / 300$,

$373 a=0.152 \mathrm{~m}, s=0.0147 \mathrm{~m}, \varphi_{b}=11.0 \mathrm{deg}, \quad E=200 \cdot 10^{9} \mathrm{~Pa}, \rho=7800 \mathrm{~kg} / \mathrm{m}^{3}$,

$374 v=0.3, \lambda=6$. The natural frequency of the first axisymmetric mode, $\omega_{1,0}$, is

375 considered for the time nondimensionalization, as already stated in (18), and a

376 modal damping factor $\varsigma_{j}=0.012, j=1,2, \ldots N_{\text {dofs }}$ is assumed.

377 A static pressure load $p_{s}=-0.40 \cdot p_{c r}$ (lower than the critical buckling pressure)

378 acts on the shell while a dynamic component, of amplitude $p_{d}=0.020 \cdot p_{c r}$, is

379 superimposed to the static one.

380 The set of nonlinear ODEs (17) is numerically solved by using the Fortran routine

381 for time integration RADAU5 [46]. This integrator was developed for solving stiff

382 ODEs and is based on the implicit Runge-Kutta method of order 5, 3-stages, with 
383 step-size control. To carry out the bifurcation analysis, the excitation frequency is 384 varied forward and backward in the frequency range $\Omega / \omega_{1,0} \in[1.07400,1.10500]$,

385 where the occurrence of dynamic instabilities were proven through a path386 following analysis in Ref. [32].

387 The parameters used for setting the time-response analysis are the following: 125

388 excitation frequency steps with a step-size of $\Delta \Omega / \omega_{1,0}= \pm 0.00025$; a sampling

389 frequency equal to 40 times the excitation frequency; 600 excitation periods of 390 integration, where only 300 periods are retained for getting rid of the transient 391 response. When the simulation starts, homogeneous initial conditions are 392 considered, then, for further steps (different frequencies), the initial conditions are 393 assumed to be the final state of the previous step, with a perturbation of amplitude 3940.01 (dimensionless) applied to every generalized coordinate. For the frequencies 395 where the system is sensitive to small perturbations and prone to exhibits chaotic 396 motion, the perturbation allows the system to leave an almost unstable orbit and 397 find remote attractors.

398 In Fig. 2(a,b), the frequency-response curves obtained by directly integrating the 399 ODEs are compared to continuation method results [32]. Starting from $400 \Omega / \omega_{1,0}=1.0740$ and considering an increasing forcing frequency (red asterisks), $401 f_{w, 1,0}$ follows the stable solution path 1 (continuous black line) and switch on 402 branch 2 after the period-doubling (PD) at $\Omega / \omega_{1,0}=1.08275$, see Fig. 2(a), where 403 the bifurcation leads to the onset of asymmetric oscillations, see Fig. 2(b). Large 404 amplitude vibrations, with a discontinuous amplitude variation, occur for $405 \Omega / \omega_{1,0} \in[1.08425,1.09375]$, where the path following analysis pointed out the 406 coexistence of multiple unstable solution (dotted black line), i.e. one or more 407 Floquet multipliers fall outside the unit circle. By considering $\Omega / \omega_{1,0}>1.09500$, $408 f_{w, 1,0}$ lies again on a stable periodic solution, while $f_{w, 1,2}^{(d)}$ follows a branch not 409 shown in Ref. [32] and asymmetric oscillations persists until a second PD 410 bifurcation at $\Omega / \omega_{1,0}=1.10125$.

411 Considering now a backward frequency variation (blue circles), the frequency412 response curve trend is almost the same obtained by considering an upward 413 frequency variation. However, when the harmonic pressure acts on the structure 414 with a frequency $\Omega / \omega_{1,0} \in[1.07650,1.08400]$, both the coordinates $f_{w, 1,0}$ and $f_{w, 1,2}^{(d)}$ 
415 follows secondary solution branches not shown by the path following analysis 416 [32]. A further reduction of the forcing frequency, leads to a sudden response 417 jump that restores a purely axisymmetric overall motion of the cap.

(a)

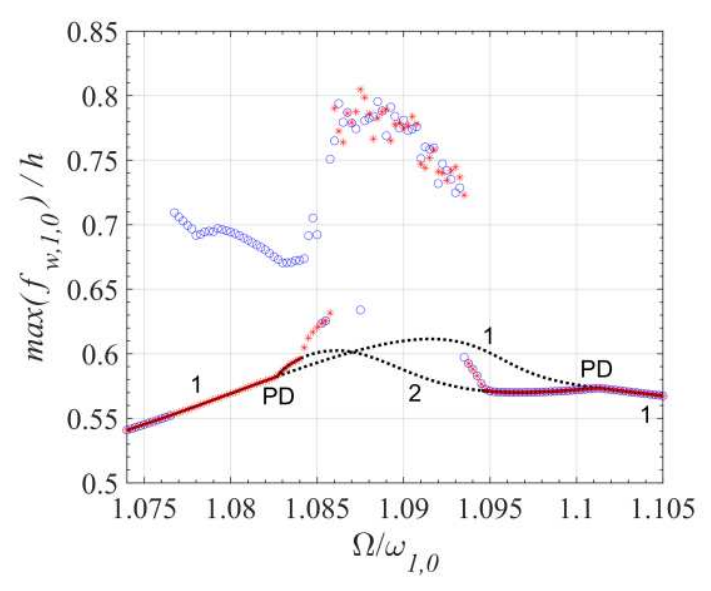

(b)

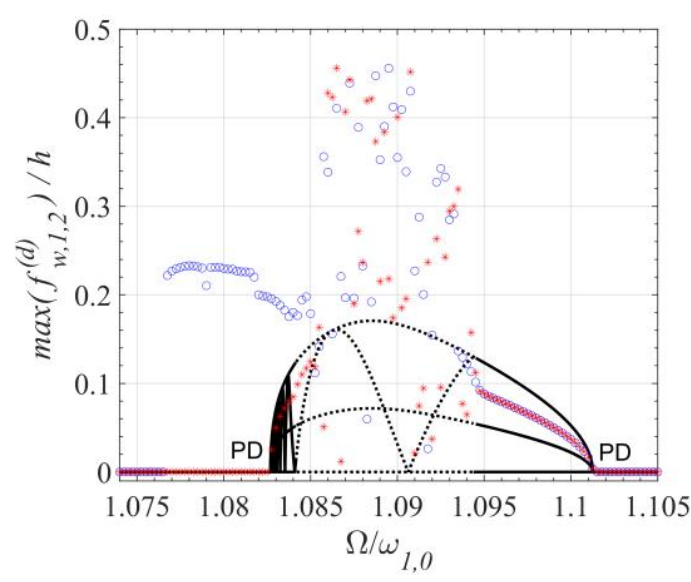

419 Fig. 2. Frequency-response curves: (a) first axisymmetric mode, (b) driven asymmetric mode 420 (1,2). (- Ref.[32], * upward frequency variation, ○ downward frequency variation, "PD" 421 period-doubling). 
423 In order to provide further information for understanding the path-following 424 analysis results, bifurcation diagrams of the Poincaré maps are here presented and 425 discussed.

426 In Fig. 3(a-d), the bifurcation diagrams obtained for an increasing excitation 427 frequency are shown. The response is fully axisymmetric until $\Omega / \omega_{1,0}=1.08275$, 428 where the activation of the asymmetric conjugate modes $f_{w, 1,2}^{(d)}$ and $f_{w, 1,2}^{(c)}$ is 429 governed by 2-T subharmonic responses, see Fig. 3(c,d), although the 430 axisymmetric generalized coordinates $f_{w, 1,0}$ and $f_{w, 2,0}$ retain $1-\mathrm{T}$ periodic 431 oscillations, Fig. 3(a, b). Nonperiodic vibrations arise for $\Omega / \omega_{1,0}=1.08425$, where 432 a Neimark-Sacker bifurcation leads to amplitude-modulated oscillations. For $433 \Omega / \omega_{1,0}=1.08600$, the quasi-periodic response collapse on a chaotic attractor.

434 Chaotic region holds until $\Omega / \omega_{1,0}=1.94250$, where quasi-periodic motion is 435 restored and the conjugated asymmetric coordinates $f_{w, 1,2}^{(d)}$ and $f_{w, 1,2}^{(c)}$ display 2-T 436 periodic oscillations. An additional excitation frequency increment gives rise to a 437 period-doubling bifurcation at $\Omega / \omega_{1,0}=1.10125$, in agreement with the finding of 438 [32]. Beyond the period doubling, the response becomes periodic with the same 439 frequency of the excitation and the contribution of the asymmetric modes on the 440 overall oscillation becomes null, as already pointed out form the analysis of the 441 frequency-response diagrams in Fig. 2(a,b).

442 Bifurcation diagrams of the Poincaré sections are now analyzed by considering a 443 decreasing excitation frequency, Fig. 4(a-d).

444 Starting from $\Omega / \omega_{1,0}=1.10500$, the structural response undergoes sequentially to 445 a period-doubling bifurcation at $\Omega / \omega_{1,0}=1.10125$ and a Neimark-Sacker 446 bifurcation at $\Omega / \omega_{1,0}=1.09450$. The amplitude-modulated oscillations burst into a 447 chaotic attractor at $\Omega / \omega_{1,0}=1.09325$. Inside the range $448 \Omega / \omega_{1,0} \in[1.08500,1.09325]$, the response jumps from chaotic to quasi-periodic 449 attractors. A further reduction of the control parameter leads to a complex 450 dynamic behavior, where the solution alternates quasi-periodic to 5T-subharmonic 451 vibrations. Then, when $\Omega / \omega_{1,0}<1.07650$, only axisymmetric states exist. 
(a)

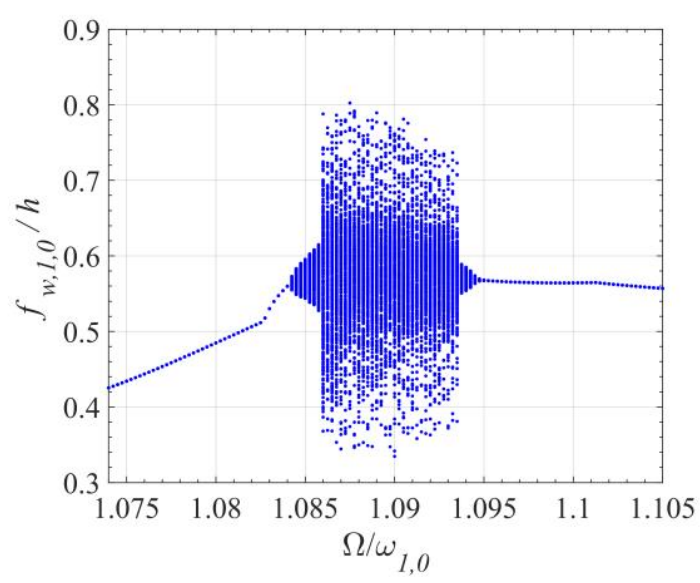

(c)

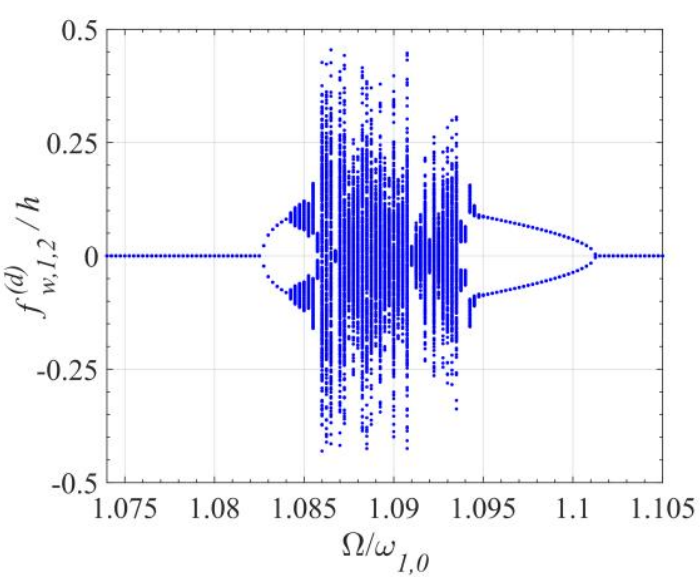

(b)

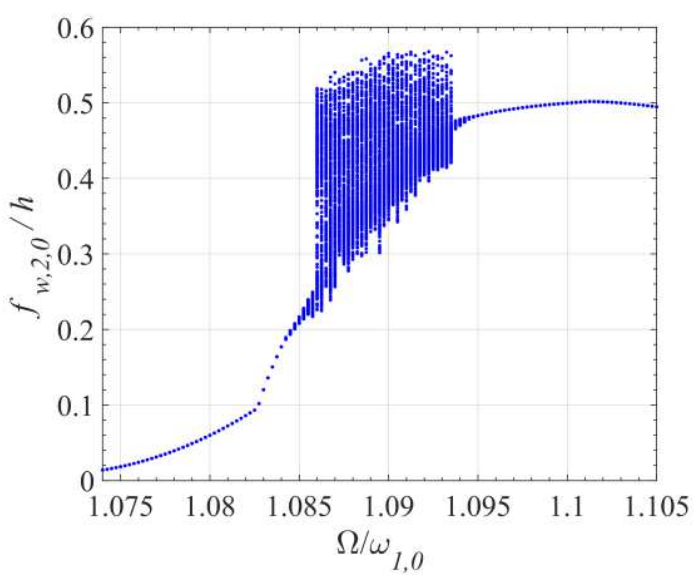

(d)

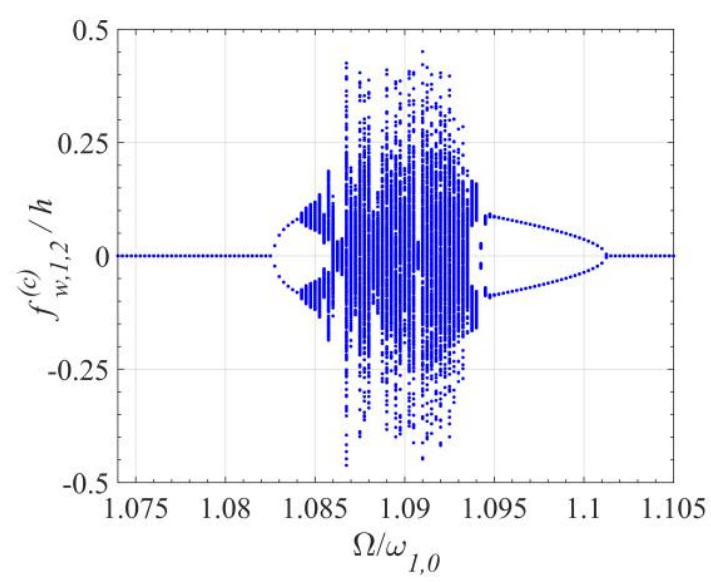

454 Fig. 3 - Bifurcation diagrams of the Poincaré section for an increasing excitation frequency:

455 (a) first axisymmetric mode, (b) second axisymmetric mode, (c) driven, and (d) companion 456 asymmetric modes (1,2).

458 From the analysis of the bifurcation diagrams, an interesting phenomenon has 459 been pointed out: for some values of the forcing frequency, axisymmetric 460 vibrations are periodic with the same frequency of the harmonic pressure, while 461 the asymmetric oscillations are 2-T subharmonic. By analyzing the set of the 462 equations, one could see that a coupling between linear terms of the coordinate $463 f_{w, 1,0}$ and $f_{w, 1,2}^{(d)}$ is missing in the first equation of the ODEs (when a perfect 464 structure is considered). On the other hand, only odd powers of $f_{w, 1,2}^{(d)}$ and products 465 between linear power of $f_{w, 1,0}$ and $f_{w, 1,2}^{(d)}$ appear in the second equation; therefore, 466 an autoparametric instability takes place when the axisymmetric mode $(1,0)$ 
467 vibrates at the same frequency of the asymmetric mode $(1,2)$, indeed, from Fig.s

$4683-4$, a period-doubling occurs when $\Omega / \omega_{1,0}=1.10125$, i.e. $\Omega / \omega_{1,2}=0.9964$.

469

(a)

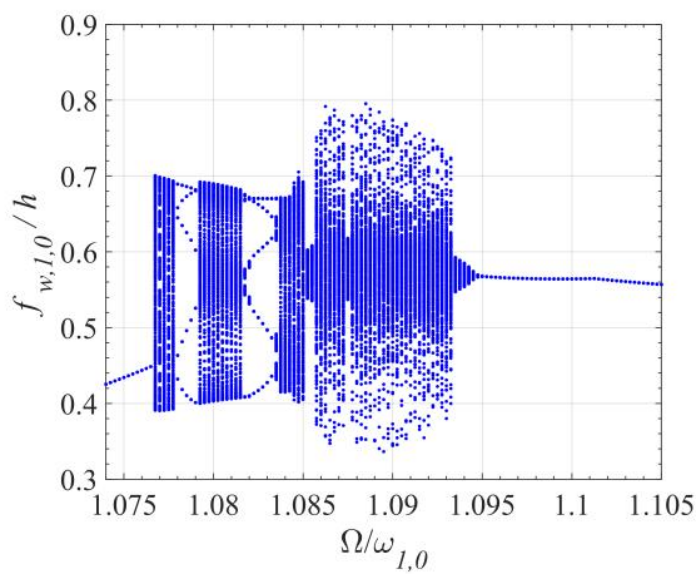

(c)

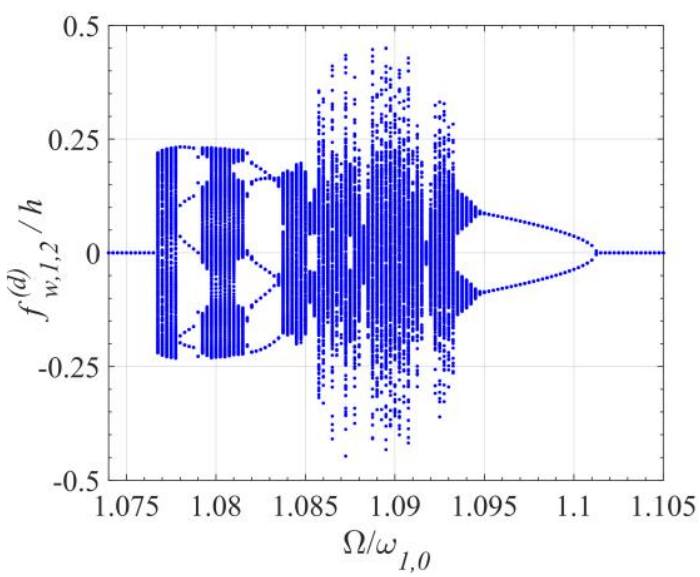

(b)

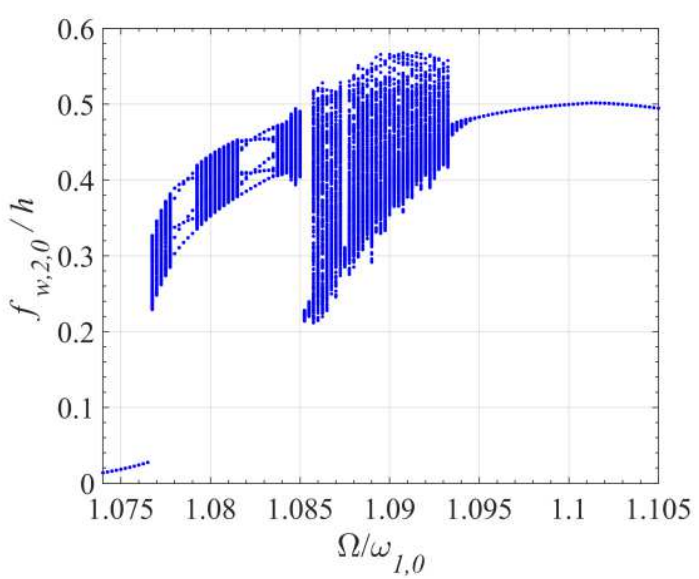

(d)

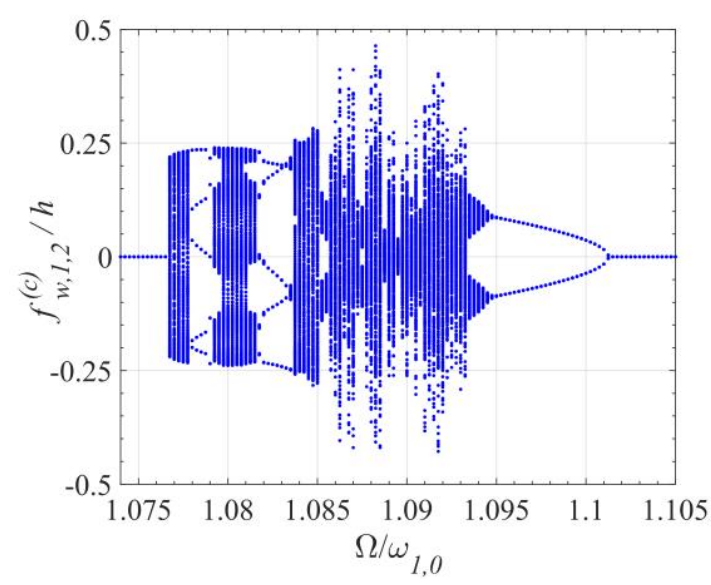

470 Fig. 4. Bifurcation diagrams of the Poincaré section for a decreasing excitation frequency:

471 (a) first axisymmetric mode, (b) second axisymmetric mode, (c) driven, and (d) companion

472 asymmetric modes $(\mathbf{1 , 2})$.

473

474 As suggested by Moon [47], in order to detect non-periodic or chaotic oscillations

475 it is not sufficient considering only frequency-response or bifurcation diagrams.

476 To this end, other mathematical tools deserve to be simultaneously considered,

477 e.g. time histories, Fourier's spectra, Poincaré sections, and phase portraits.

478 Without loss of generality, only the case of decreasing excitation frequency is 479 here deeply investigated. 
(a)

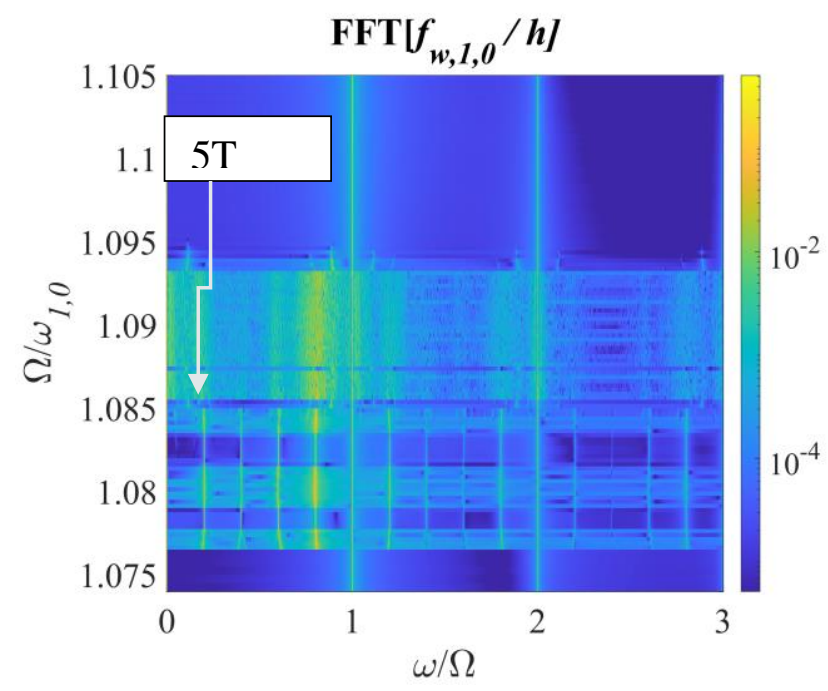

(b)

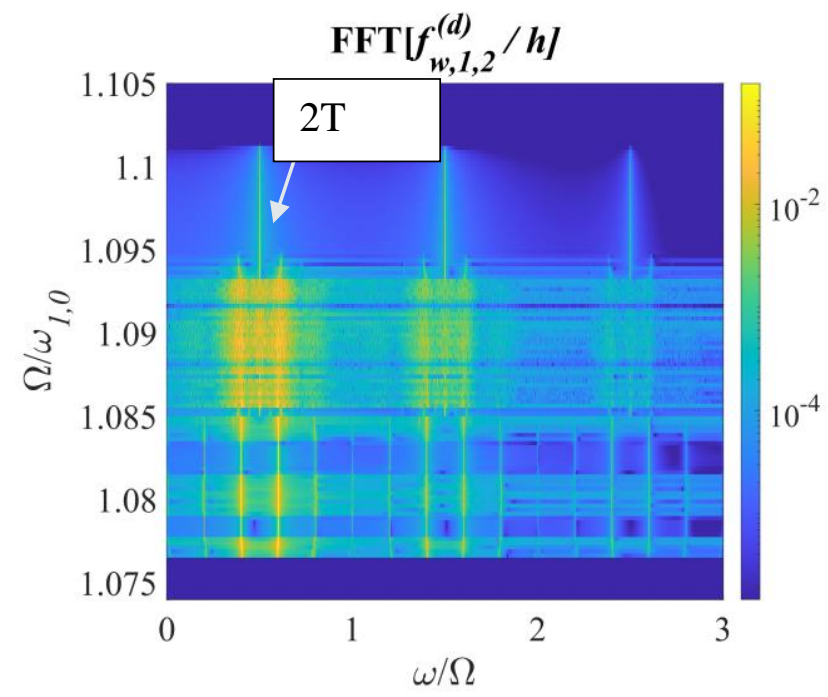

481 Fig. 5 - Spectrograms of the modal coordinates for a decreasing excitation frequency: (a)

482 first axisymmetric mode, (b) driven companion asymmetric modes $(1,2)$.

484 In Fig. 5(a) the spectrogram of $f_{w, 1,0}$ is shown. The energy content is localized at 485 the same frequency of the excitation until the instability onset, where the energy 486 spreads on a broad frequency range. On the other hand, the response of the 487 asymmetric mode $f_{w, 1,2}^{(d)}$ is mainly 1/2-subharmonic, see Fig. 5(b). When the 488 frequency of the harmonic pressure is decreasing and crosses $\Omega / \omega_{1,0}=1.0850$, 489 5T-subharmonic components of the response are clearly visible from both spectra. 490 It is worthwhile to note that in the spectrum of $f_{w, 1,2}^{(d)}$ the main 1T-harmonic is 491 almost absent except in the frequency range of strong subharmonic vibrations. 
492 In the following, the development of chaotic oscillations is shown and the 493 behavior of the driven asymmetric mode $f_{w, 1,2}^{(d)}$ is deeply addressed to complete the 494 description of the dynamic scenario.

495 In Fig. 6(a-d) the case $\Omega / \omega_{1,0}=1.0970$ is discussed. The driven asymmetric mode $496 f_{w, 1,2}^{(d)}$ shows a $1 / 2$-subharmonic: only odd harmonics appear in the spectrum 497 because of the symmetry of the time waveform, Fig. 6(a,b); two points are present 498 in the Poincaré map, Fig. 6(c); the regular limit-cycle shown by the phase portrait 499 confirms the periodicity of the vibration, Fig. 6(d).

$$
\Omega / \omega_{1,0}=1.0970
$$

(a)

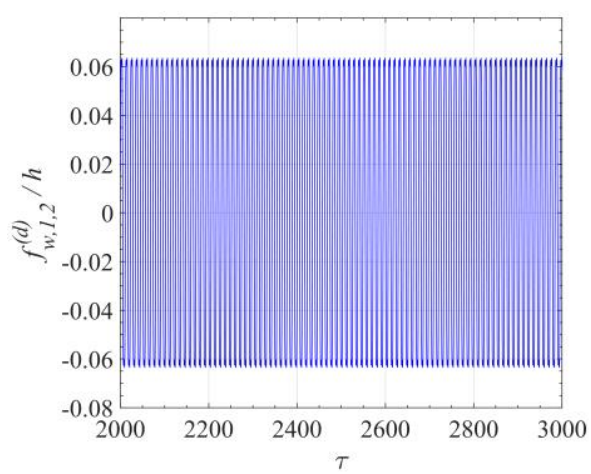

(c)

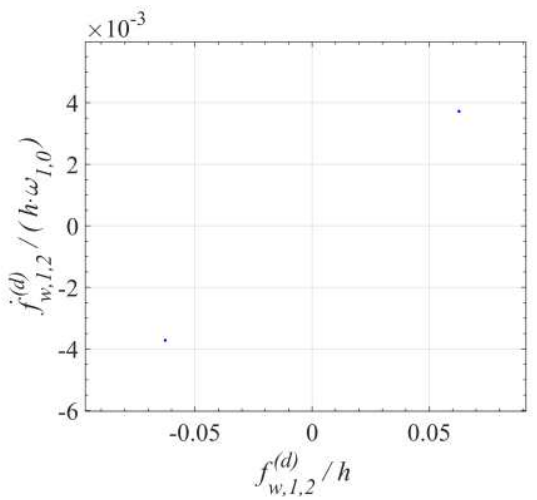

(b)

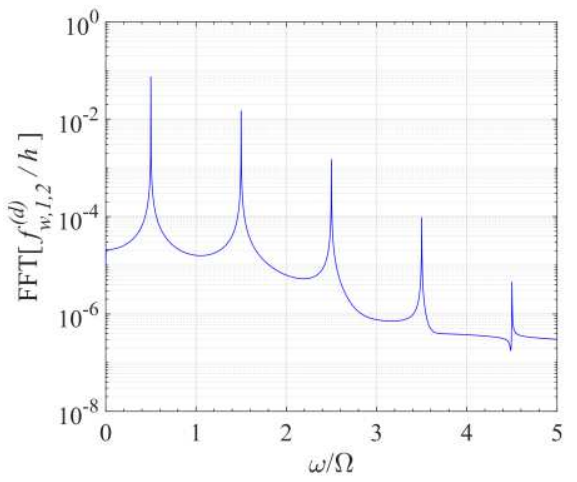

(d)

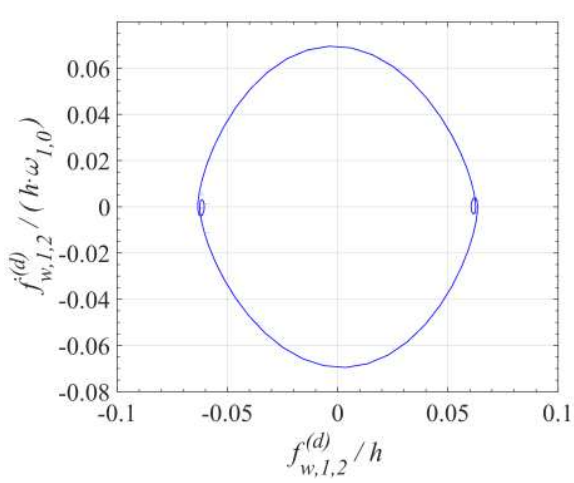

Fig. 6. 2T subharmonic response of the driven modal coordinate $(1,2)$. Decreasing excitation frequency. (a) Time history, (b) Fourier spectrum, (c) Poincaré map, and (d) phase portrait.

504 The forcing frequency is now reduced to $\Omega / \omega_{1,0}=1.09375$, and the system is in 505 the un-steady region, as depicted in Fig. 4. The Neimark-Sacker bifurcation gives 506 rise to quasi-periodic oscillations, thus the response can be seen as a sum of many 507 periodic functions, where two or more frequencies are incommensurate [48]: in 
508 this case the time response is amplitude-modulated, Fig. 7(a); the carrier

509 frequency is $\omega / \Omega=1 / 2$ and sidebands (modulation frequency $\Delta \omega / \Omega=0.11$ ) are

510 present, Fig. 7(b); the Poincaré map displays two closed non-connected sets,

511 therefore the response is 2-period quasiperiodic with modulation of the amplitude 512 [49], and the orbit does not close on itself, Fig. 7(c,d).

513

$$
\Omega / \omega_{1,0}=1.09375
$$

(a)

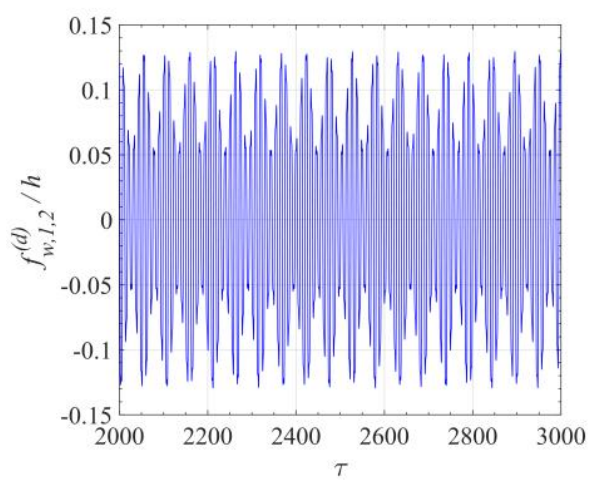

(c)

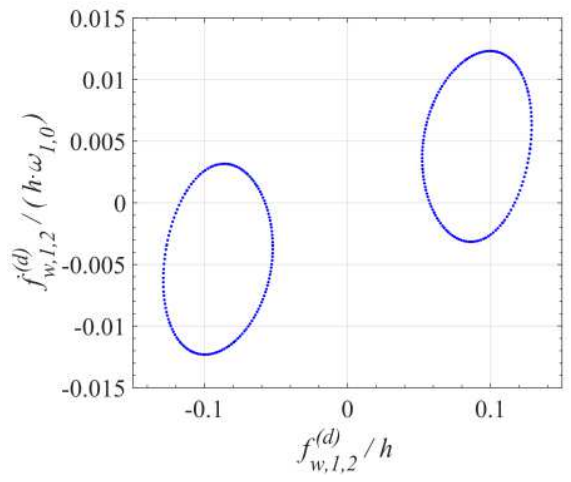

(b)

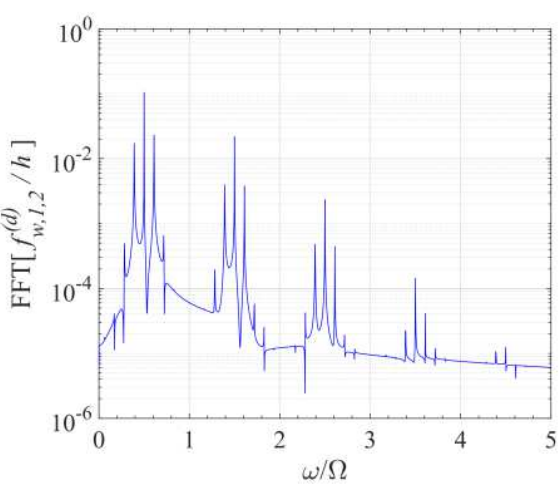

(d)

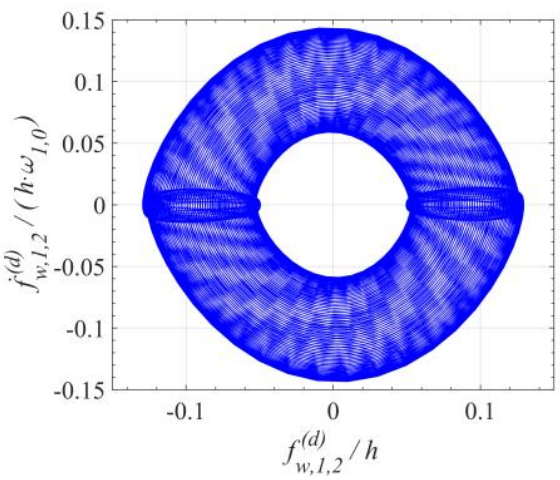

514 Fig. 7. Amplitude-modulated response of the driven modal coordinate $(1,2)$. Decreasing 515 excitation frequency. (a) Time history, (b) Fourier spectrum, (c) Poincaré map, and (d) phase 516 portrait. 
519 The case at $\Omega / \omega_{1,0}=1.0900$, is now analyzed. Chaotic vibrations can be observed:

520 the time history exhibits intermittency of the response bursts, Fig. 8(a); the

521 spectrum is characterized by a spreading of energy over a broad-band around the

522 carrier frequency (and multiples) $\omega / \Omega=1 / 2$, Fig. 8(b); the Poincare section

523 shows a set of randomly distributed points, where the dimension of the set does

524 not appear integer, Fig. 8(c), and the trajectory is completely irregular, Fig. 8(d).

$$
\Omega / \omega_{1,0}=1.0900
$$

(a)

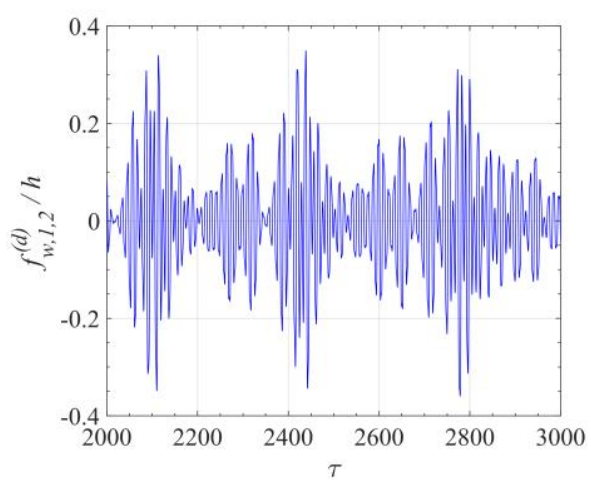

(c)

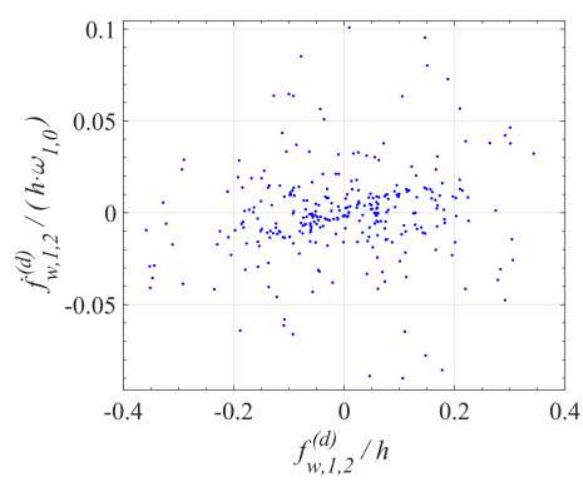

(b)

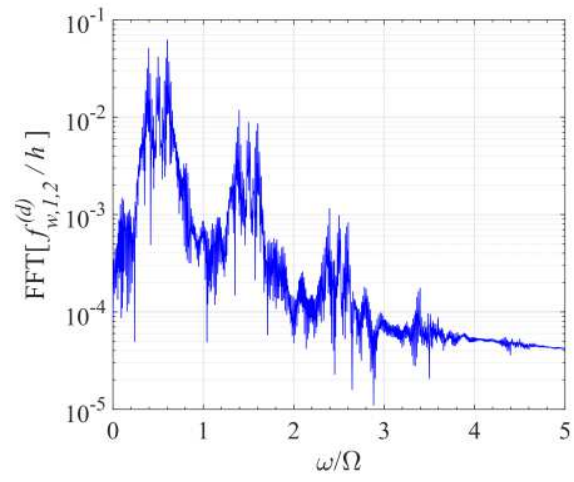

(d)

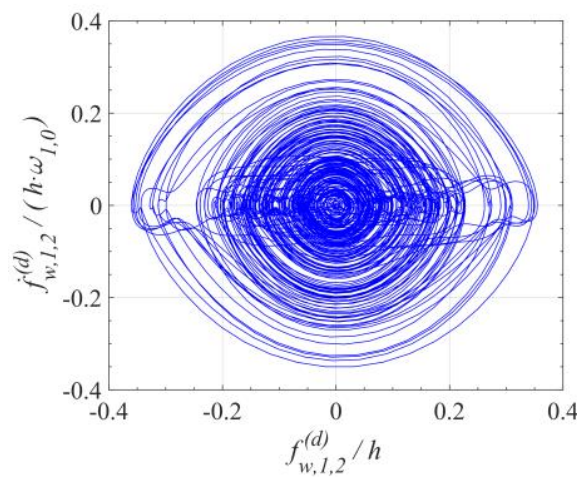

Fig. 8. Chaotic response of the driven modal coordinate $(1,2)$. Decreasing excitation frequency. (a) Time history, (b) Fourier spectrum, (c) Poincaré map, and (d) phase portrait. 
530 Maps of chaotic motion need a larger number of points. Therefore, an additional

531 Poincaré section obtained by considering 10000 forcing periods is shown in Fig.

532 9. This map clearly shows chaotically modulated oscillations (weak chaos): the

533 central dense pattern is due to the high-frequency vibration, while the outer sparse

534 region is caused by intermittent bursts governed by a slow dynamic. Such set

535 distribution is justified by the Fourier spectrum where, despite its broad energy

536 distribution, the subharmonic components and sidebands give a significant

537 contribution to the overall dynamic of the asymmetric modal coordinate.

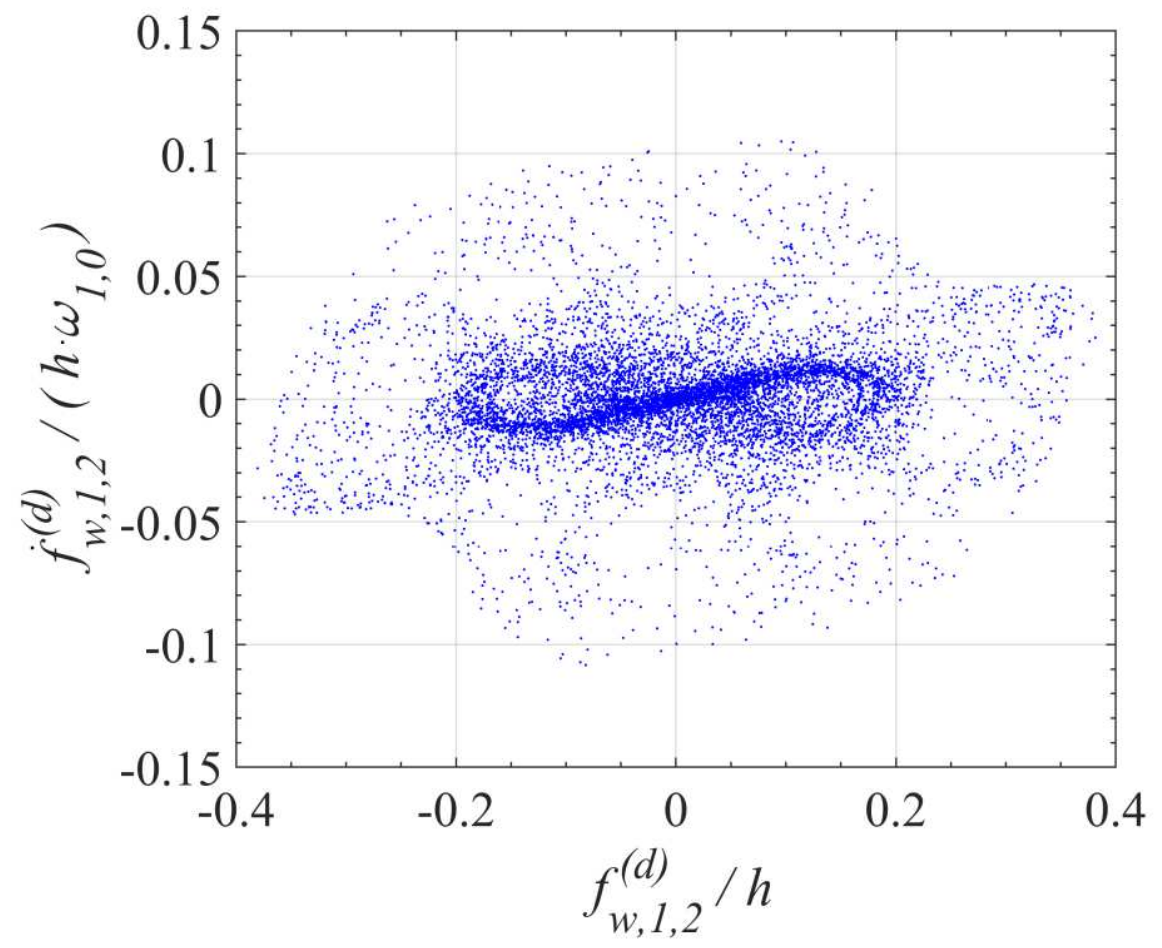

539 Fig. 9 - Poincaré map of chaotically modulated oscillations.

541 After a further reduction of the excitation frequency, the system exits form the 542 chaotic region even though it is still inside the "instability region", where an 543 alternance of periodic and non-periodic regions is present. More specifically, the 544 case $\Omega / \omega_{1,0}=1.0827$ is now analyzed. Here the cap response becomes $5-\mathrm{T}$ 545 subharmonic: the time history appears asymptotically stable, Fig. 10(a); the 546 fundamental frequency is $\omega / \Omega=1 / 5$, Fig. 10(b); the Poincaré map shows five 547 dots, Fig. 10(c); the solution follows a closed regular orbit, Fig. 10(d). 


$$
\Omega / \omega_{1,0}=1.0827
$$

(a)

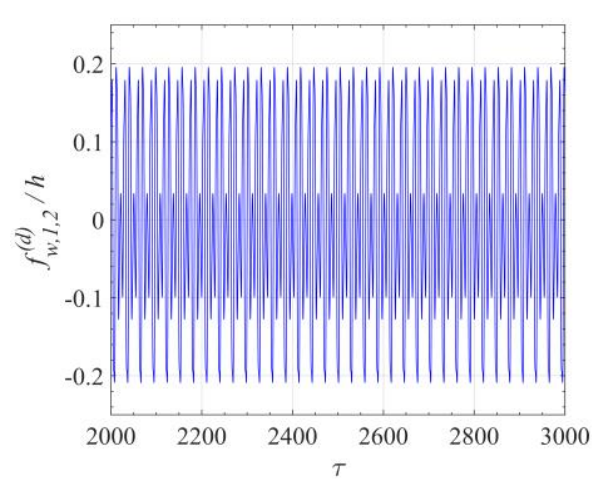

(c)

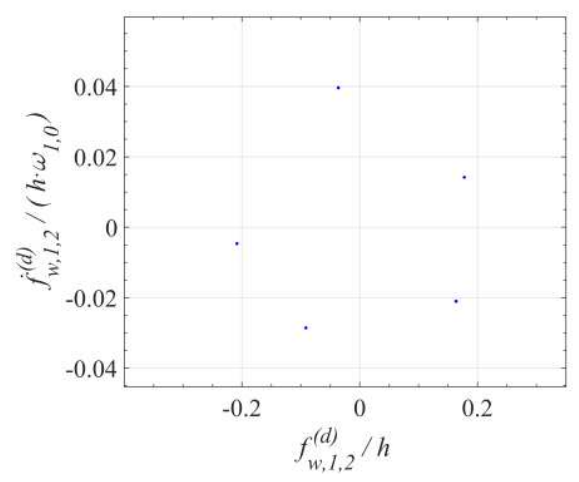

(b)

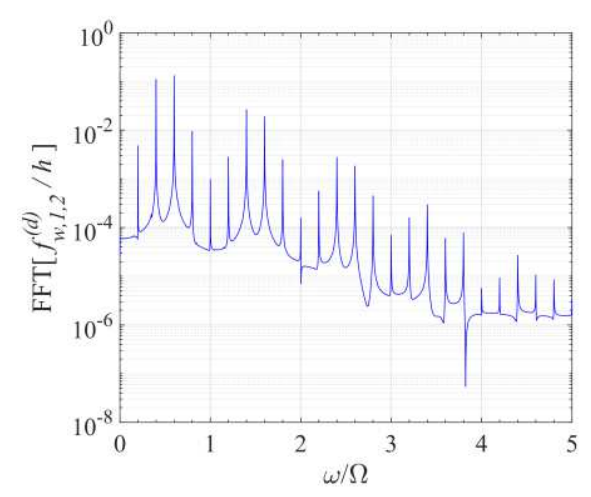

(d)

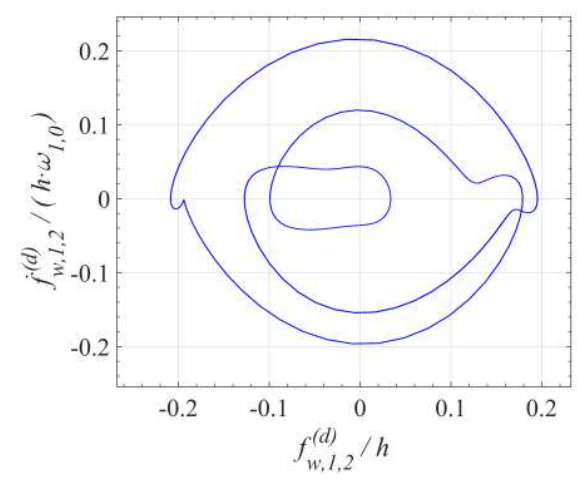

550 Fig. 10. 5T-subharmonic response of the driven modal coordinate $(1,2)$. Decreasing 551 excitation frequency. (a) Time history, (b) Fourier spectrum, (c) Poincaré map, and (d) phase 552 portrait.

554 The last case to be investigated is $\Omega / \omega_{1,0}=1.07735$. The coordinate $f_{w, 1,2}^{(d)}$ exhibits 555 quasi-periodic oscillations, where the superposition of several periodic functions 556 can be noted by simply observing the time history, Fig. 11(a). The vibration is 557 strongly characterized by a 1/5-subharmonic contribution Fig. 11(b); the phase 558 portrait and the Poincare section confirms the character of the response, Fig. 559 11(c,d). As already shown by the frequency-response curves and the bifurcation 560 diagrams, a further decrease in the excitation frequency restores a periodic 561 oscillation with a null contribution of the non-symmetric modes. 


$$
\Omega / \omega_{1,0}=1.07735
$$

(a)

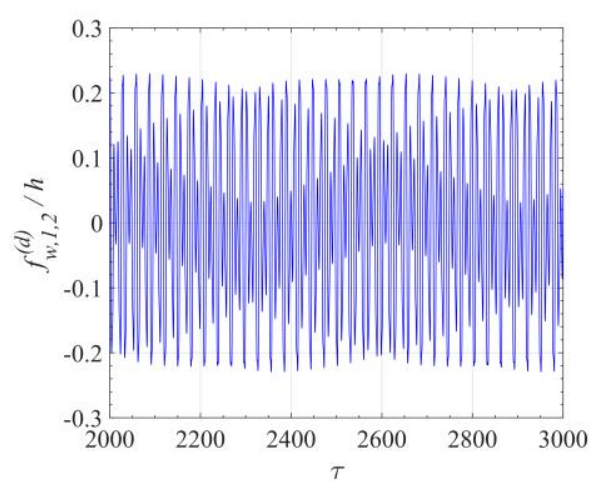

(c)

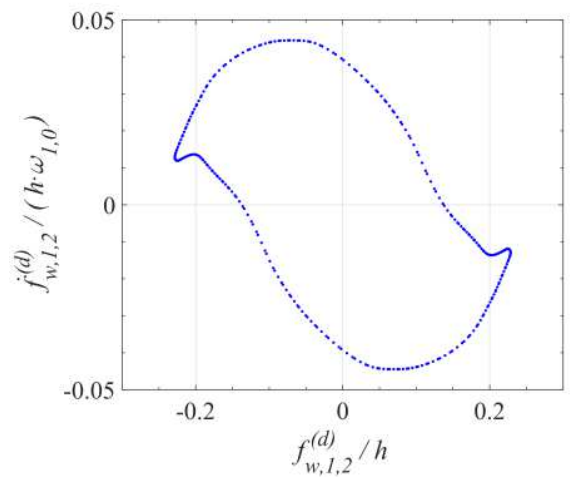

(b)

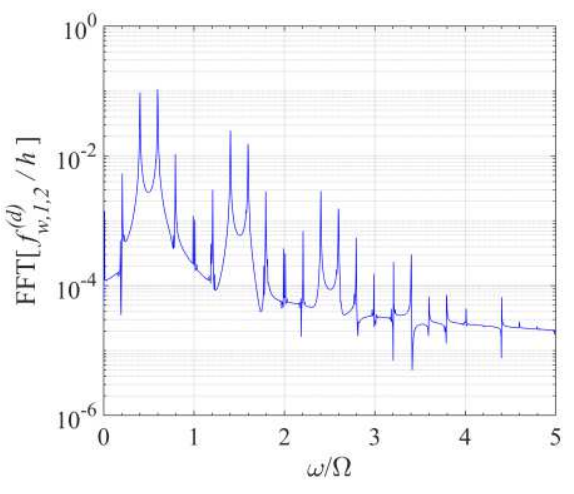

(d)

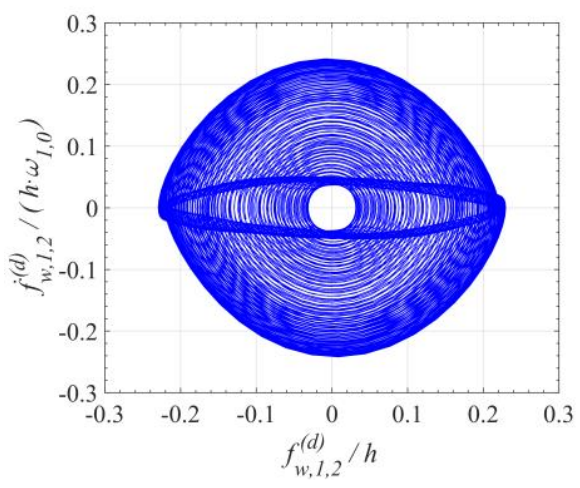

564 Fig. 11. Quasi-periodic response of the driven modal coordinate (1,2). Decreasing excitation 565 frequency. (a) Time history, (b) Fourier spectrum, (c) Poincaré map, and (d) phase portrait..

\section{Conclusions}

568 The problem of a shallow spherical cap exhibiting asymmetric oscillations when

569 subjected to a uniform harmonic pressure has been investigated. The

570 Novozhilov's nonlinear shell theory has been considered for defining the strain-

571 displacement relations. The partial differential equations are reduced to a finite

572 dimension by using an energy formulation based on Rayleigh-Ritz approach and

573 Lagrange equations. For describing the cap deformation, the set of displacement

574 field trial functions have been expressed by means of Legendre polynomials and

575 trigonometric functions. A static compressive pressure has been superimposed to

576 a harmonic one. Bifurcation diagrams are investigated against the excitation

577 frequency. The dynamic scenario shows that the spherical cap vibrations turned 
578 out to be often asymmetric, non-periodic, with multiple jumps among

579 subharmonic, quasi-periodic, and chaotic vibrations.

581 Acknowledgements

582 The authors acknowledge the University of Modena and Reggio Emilia for

583 supporting this research through the project "Interflu / Non-Newtonian Fluids and

584 Fluid-Structure Interaction".

586 Funding

587 FAR2020 Mission Oriented - (CUP E99C20001160007).

589 Conflict of Interests

590 The authors declare they have no conflict of interests.

\section{Data availability}

593 Data are available from the authors upon reasonable request.

\section{References}

596 [1] Krenzke, M. A., and Kiernan, T. J., 1963, "Elastic Stability of Near-Perfect Shallow

Spherical Shells," AIAA J., 1(12), pp. 2855-2857. Huang, N., 1964, “Unsymmetrical Buckling of Thin Shallow Spherical Shells,” J. Appl. Mech., 31(3), pp. 447-457.

602 [4] Yamada, S., Uchiyama, K., and Yamada, M., 1983, "Experimental Investigation of the

[3] Weinitschke, H. J., 1965, “On Asymmetric Buckling of Shallow Spherical Shells,” J.

604 [5] Hutchinson, J. W., 1965, Imperfection-Sensitivity of Externally Pressurized Spherical

605 Shells, National Aeronautics and Space Administration, NASA-CR-68613.

606 [6] Gonçalves, P. B., and Croll, J. G. A., 1992, “Axisymmetric Buckling of Pressure-Loaded 607 Spherical Caps,” J. Struct. Eng., 118(4), pp. 970-985.

608 [7] NASA, 1969, Buckling of Thin Walled Doubly-Curved Shells, National Aeronautics and 
610 [8] Wagner, H. N. R., Hühne, C., and Niemann, S., 2018, "Robust Knockdown Factors for the Design of Spherical Shells under External Pressure: Development and Validation,” Int. J. Mech. Sci., 141(January), pp. 58-77.

613 [9] Evkin, A. Y., and Lykhachova, O. V, 2019, "Design Buckling Pressure for Thin Spherical Shells: Development and Validation,” Int. J. Solids Struct., 156-157, pp. 61-72.

615 [10] Lock, M. H., Okubo, S., and Whittier, J. S., 1968, "Experiments on the Snapping of a

[11] Stricklin, J. A., Haisler, W. E., Macdougall, H. R., and Stebbins, F. J., 1968, "Nonlinear

[12] Stricklin, J. A., Martinez, J. E., Tillerson, J. R., Hong, J. H., and Haisler, W. E., 1971,

[13] Huang, N. C., 1969, “Axisymmetric Dynamic Snap-through of Elastic Clamped Shallow

[16] Akkas, N., 1976, "Bifurcation and Snap-through Phenomena in Asymmetric Dynamic

[17] Kao, R., and Perrone, N., 1971, “Asymmetric Buckling of Spherical Caps With

[18] Kao, R., 1980, "Large Deformation Elastic-Plastic Buckling Analysis of Spherical Caps

[19] Kao, R., 1980, “Nonlinear Dynamic Buckling of Spherical Caps with Initial

[20] Yu, Y. Y., 1964, “Generalized Hamilton's Principle and Variational Equation of Motion in

[22] Evensen, H. A., and Evan-Iwanowski, R. M., 1967, "Dynamic Response and Stability of Nonlinear Elasticity Theory, with Application to Plate Theory,” J. Acoust. Soc. Am., 36(1), pp. 111-120.

[21] Grossman, P. L., Koplik, B., and Yu, Y., 1969, "Nonlinear Vibrations of Shallow Spherical Shells,” J. Appl. Mech., 36(3), pp. 451-458. Shallow Spherical Shells Subject to Time-Dependent Loading.," AIAA J., 5(5), pp. 969976.

[23] Yasuda, K., and Kushida, G., 1984, "Nonlinear Forced Oscillations of a Shallow Spherical Shell,” Bull. JSME, 27(232), pp. 2233-2240.

[24] Gonçalves, P. B., 1994, “Axisymmetric Vibrations of Imperfect Shallow Spherical Caps Under Pressure Loading,” J. Sound Vib., 174(2), pp. 249-260.

[25] Gonçalves, P. B., 1993, “Jump Phenomena, Bifurcations, and Chaos in a Pressure Loaded Spherical Cap Under Harmonic Excitation,” Appl. Mech. Rev., 46(11S), pp. S279-S288. 
[26] Soliman, M. S., and Goncalves, P. B., 2003, "Chaotic Behavior Resulting in Transient and Steady State Instabilities of Pressure-Loaded Shallow Spherical Shells,” J. Sound Vib.,

[27] Thomas, O., Touzé, C., and Chaigne, A., 2005, "Non-Linear Vibrations of Free-Edge Thin

[29] Touzé, C., and Thomas, O., 2006, "Non-Linear Behaviour of Free-Edge Shallow Spherical Shells: Effect of the Geometry,” Int. J. Non. Linear. Mech., 41(5), pp. 678-692.

[31] Krysko, V. A., Awrejcewicz, J., Dobriyan, V., Papkova, I. V., and Krysko, V. A., 2019,

[33] Novozhilov, V. V., 1953, Foundations of the Nonlinear Theory of Elasticity, Graylock

[34] Leissa, A. W., 1973, Vibration of Shells, National Aeronautics and Space Administration,

[35] de Souza, V. C. M., and Croll, J. G. A., 1980, “An Energy Analysis of the Free Vibrations

[36] Leissa, A. W., 2005, "The Historical Bases of the Rayleigh and Ritz Methods," J. Sound Vib., 287(4-5), pp. 961-978.

[37] Meirovitch, L., 1942, “Fundamentals of Vibration Study,” Nature, 150(3805), pp. 392392.

[38] Evensen, D. A., 1966, “Nonlinear Flexural Vibrations of Thin Circular Rings,” J. Appl.

[39] Kubenko, V. D., Koval'chuk, P. S., and Krasnopol'skaya, T. S., 1982, "Effect of Initial Camber on Natural Nonlinear Vibrations of Cylindrical Shells," Sov. Appl. Mech., 18(1), pp. 34-39.

[40] Amabili, M., Pellicano, F., and Païdoussis, M. P., 1999, "Non-Linear Dynamics and Stability of Circular Cylindrical Shells Containing Flowing Fluid. Part I: Stability,” J. Sound Vib., 225(4), pp. 655-699.

[41] Amabili, M., Pellicano, F., and Païdoussis, M. P., 2000, "Non-Linear Dynamics and Stability of Circular Cylindrical Shells Containing Flowing Fluid. Part III: Truncation Effect without Flow and Experiments," J. Sound Vib., 237(4), pp. 617-640.

[42] Amabili, M., 2008, Nonlinear Vibrations and Stability of Shells and Plates, Cambridge 
University Press, Cambridge.

693 [43] Pellicano, F., 2007, "Vibrations of Circular Cylindrical Shells: Theory and Experiments,"

$694 \quad$ J. Sound Vib., 303(1-2), pp. 154-170.

695 [44] Amabili, M., and Breslavsky, I. D., 2015, "Displacement Dependent Pressure Load for Finite Deflection of Doubly-Curved Thick Shells and Plates,” Int. J. Non. Linear. Mech.,

698 [45] Zoelly, R., 1915, “Uber Ein Knickungsproblem an Der Kugelschale.," ETH Zürich, 699 Zürich, Switzerland.

700 [46] Hairer, E., and Wanner, G., 1996, Solving Ordinary Differential Equations II, Springer,

701

702 Berlin.

[47] Moon, F. C., 2004, Chaotic Vibrations : An Introduction for Applied Scientists and Engineers, Wiley-Interscience, New York.

[48] Parker, T. S., and Chua, L. O., 1987, "Chaos: A Tutorial for Engineers,” Proc. IEEE, 75(8), pp. $982-1008$.

706 [49] Nayfeh, A. H., and Balachandran, B., 1995, Applied Nonlinear Dynamics, Wiley, Weinheim.

708 